\title{
Sobre a Infinidade dos Primos, o Princípio da Casa dos Pombos e a Função Exponencial na Aritmética Limitada
}

\author{
Rafael Russo Setin Orientador: Ricardo Bianconi
}




\section{Resumo}

Estudaremos aqui problemas de demonstrações em teorias fracas da Aritmética Limitada os teoremas sobre a infinidade dos primos e do princípio da casa dos pombos (limitado). Serão dadas soluções parciais para os dois problemas anteriores, além da demonstração que o grafíco de $x^{y}=z$ tem definição $\Delta_{0}$. 


\section{Abstract}

We study here the problems of proving in weak theories of Bounded Arithmetic the theorems about the existence of arbitrarily large prime numbers and the (limited) pigeon hole principle. We will give partial solutions for the two foregoing problems, and the proof that the graphic of $x^{y}=z$ has a $\Delta_{0}$ definition. 


\section{Agradecimentos}

Queria agradecer acima de tudo meu orientador, Prof.Doutor Ricardo Bianconi, pela ajuda inestimável sem a qual eu não conseguiria produzir essa dissertação. A seguir gostaria de agradecer diversos Professores Doutores que me incentivaram e ajudaram muito durante a graduação e o mestrado, entre eles Paulo F. Leite, Zara I. Abud, Rosa Maria S. B. Chaves, Lucilia D. Borsari, Henrique S. Panzarelli e Claudia C. Candido. Por fim agradeço meus familiares e minha namorada pelo incentivo e compreensão. 


\section{Sumário}

1 Introdução 1

2 Preliminares 5

2.1 Introdução . . . . . . . . . . . . . . . . . . . . 5 5

2.2 Conceitos básicos . . . . . . . . . . . . . . . 5

2.3 Gráfico $\Delta_{0}$ da exponencial . . . . . . . . . . . . . . . . 6

2.4 Derramamento, Boa Ordem e Coleção . . . . . . . . . . . . . . . . 9

3 PHP e Primos 11

3.1 Introdução . . . . . . . . . . . . . . . . . . . 11

3.2 O Resultado Principal . . . . . . . . . . . . . . . . . . . . 12

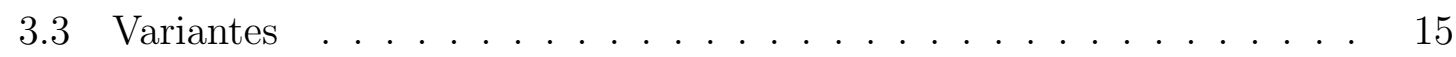

3.4 Primos e PHP ......................... 20

4 Conclusão $\quad 29$

Referências Bibliográficas $\quad 31$

Índice Remissivo $\quad 33$ 


\section{Capítulo 1}

\section{Introdução}

Uma importante contribuição que o estudo da lógica traz para outras áreas da matemática é a compreensão de qual o menor número de axiomas realmente necessários para demonstrar um dado teorema. Podemos ver uma ideia similar aplicada na Geometria e na Teoria dos Conjuntos quando tentaram, através dos outros axiomas, provar o Axioma das Paralelas (Geometria) e o Axioma da Escolha (Teoria dos Conjuntos), o que levou à prova da independência de ambos e o surgimento de novas geometrias e teorias dos conjuntos (com esses axiomas modificados ou excluídos).

Na dissertação a seguir usaremos essa ideia na Teoria dos Números, pensando em questões como: quais os axiomas necessários para provar a infinidade dos números primos? E para provar o princípio da casa dos pombos? Ou pensando de maneira "inversa": o que podemos demonstrar quando enfraquecemos a Aritmética de Peano?

Para obter essa Aritmética enfraquecida $\left(I \Delta_{0}\right)$ o Princípio da Indução Finita só poderá ser usado em fórmulas com algum tipo de limitação $\left(\Delta_{0}\right)$, e preservaremos os outros axiomas da Teoria dos Números. A escolha de $I \Delta_{0}$ se dá pela ligação deste sistema com Complexidade Computacional, $I \Delta_{0}$ está associado as funções de crescimento polinomial (uma função é dita polinomial se o tempo de execução de seu algoritmo pode ter um polinômio como limitante superior) que são facilmente calculáveis computacionalmente. Os axiomas de $I \Delta_{0}$ não conseguem provar argumentos que usam funções de crescimento não polinomial e a demonstração dada por Euclides usa o fatorial que é uma função de crescimento exponencial (não polinomial).Quando necessário iremos acrescentar a $I \Delta_{0}$ funções com taxa de crescimento diferente (subexponenciais), para tentar responder as perguntas acima. 
Isto é, iremos abordar os seguintes problemas e suas relações:

Problema 1 Podemos ter $I \Delta_{0} \vdash \forall x \exists y>x(y$ primo $)$ ?

Problema 2 Podemos ter $I \Delta_{0} \vdash \Delta_{0} P H P$ ?

A expressão $\Delta_{0}$ PHP é o Princípio da Casa dos Pombos (em inglês, Pigeon Hole Principle) aplicado a fórmulas $\Delta_{0}$, isto é, o esquema abaixo:

$$
\forall x \leq z \exists y<z \theta(x, y) \longrightarrow \exists x_{1}, x_{2} \leq z \exists y<z\left(x_{1} \neq x_{2} \wedge \theta\left(x_{1}, y\right) \wedge \theta\left(x_{2}, y\right)\right)
$$

ou de modo equivalente em $I \Delta_{0}$, para $F(x, y)$ uma fórmula $\Delta_{0}$ descrevendo uma função, escreveremos $F: a \longmapsto b$ como a função injetora de $a$ em $b$ e

$$
\neg \exists z F: z+1 \longmapsto z,
$$

significará, não existe função injetora de $z+1$ em $z$, onde $z+1=\{0,1, . ., z\}$ e $z=\{0,1, \ldots, z-1\}$.

No Capítulo 2 provaremos o Teorema do Derramamento e mostraremos, de forma mais detalhada, uma definição $\Delta_{0}$ de $x^{y}=z$ (usando como base [2]). E também demonstraremos o Teorema da Coleção e uma versão $\Delta_{0}$ do Princípio da Boa Ordem, sendo ambos mais detalhados a partir de [2]. Todos estes resultados serão utilizados no capítulo 3. No Capítulo 3, daremos soluções parciais para os problemas 1 e 2 e estudaremos as relações entre ambos de modo similar ao feito em [7] (provaremos todos os Teoremas de modo mais detalhado, com exceção do Teorema de Sylvester). No Capítulo 4, concluímos este trabalho e apresentamos problemas em aberto.

Vejamos um pouco do contexto dos nossos problemas. O problema 1 foi levantado por A. J. Wilkie em 1977 e pouco depois, em sua tese de doutorado [8], A.R. Woods deu soluções parciais para ambos os problemas e mostrou uma relação entre estes (mais avanços nesse sentido foram feitos em [7]), além da interessante conjectura

$$
I \Delta_{0}(\pi)+\operatorname{def}(\pi) \models \text { existência de primos arbitrariamente grandes }
$$

onde $\pi(x)$ é o símbolo de função cuja interpretação nos dá o número de primos menores que $x$, e $\operatorname{def}(\pi)$ é o axioma que define $\pi(x)$. Isto é, se tivermos, na Aritmética Limitada, uma função que conta os primos menores que algum número fixado devemos conseguir provar, a existência de primos arbitrariamente grandes. Progressos significativos foram feitos em relação a essa conjectura, ver [9] e [5].

A notação é a usual. Em particular todos os logaritmos estão na base 2 e em expressões como $\log (x),(\epsilon+1) x$ entre outras, vamos sempre pensar na parte inteira 
dos números. E $M$ será um modelo enumerável não standard de $I \Delta_{0}$, e seja $\Delta_{0}^{M}$ os subconjuntos de $M$ definidos por $\Delta_{0}$ fórmulas com parâmetros em $M$. 


\section{Capítulo 2}

\section{Preliminares}

\subsection{Introdução}

Aqui apresentaremos, de forma mais detalhada, uma definição $\Delta_{0}$ de $x^{y}=z$, usando a construção e as definições dadas em [2]. Além das demontrações do Teorema do Derramamento, Teorema da Coleção e uma versão $\Delta_{0}$ do Princípio da Boa Ordem, sendo os dois últimos mais detalhados a partir de [7].

\subsection{Conceitos básicos}

Lembramos algumas funções básicas e suas definições.

Seja $L=\{0,1,+, \cdot, \leq\}$ a linguagem de primeira ordem para a aritmética. Uma fórmula de $L$ é chamada $\Delta_{0}$, ou limitada, se todos os seus quantificadores ocorrem no contexto $\forall x(x \leq t(y) \longrightarrow \ldots)$ ou $\exists x(x \leq t(y) \wedge \ldots)$, abreviados por $\forall x \leq t(y) \ldots$ e $\exists x \leq t(y) \ldots$, onde $t$ é um termo em $L$. Aqui investigaremos certas extensões do sistema $I \Delta_{0}$ (indução finita aplicada em fórmulas $\Delta_{0}$ ), que é axiomatizada em $L$ pelo esquema

$$
\forall x \forall z((\phi(x, 0) \wedge \forall y \leq z(\phi(x, y) \longrightarrow \phi(x, y+1)) \longrightarrow \forall y \leq z \phi(x, y))), \phi \in \Delta_{0}
$$

(Princípio Limitado da Indução Finita) juntamente com as propriedades básicas da Aritmética: 
1. $(0 \leq 0 \wedge \neg 1 \leq 0)$

2. $\forall x(x+0=x \wedge x \cdot 0=0 \wedge x \cdot 1=x)$

3. $\forall x \forall y(x+1=y+1 \longrightarrow x=y)$

4. $\forall x \forall y(x \leq y+1 \longrightarrow(x \leq y \vee x=y+1))$

5. $\forall x \forall y(x+(y+1)=(x+y)+1)$

6. $\forall x \forall y(x(y+1)=x \cdot y+x)$

O resto da divisão de $u$ por $v$, será denotado por $r m(u, v)=z$ e é definido pela fórmula $((u=v \cdot q+z) \wedge 0 \leq z<v)$; a fórmula que define $x$ é primo é $[x>1 \wedge \forall y<$ $x \forall z<x(x=y \cdot z \rightarrow(x=y \vee x=z))]$. Assumimos conhecimento de propriedades básicas dessas e outras relações elementares e codificações de pares, sequências (via a função $\beta$ de Gödel). Para tais resultados, veja, por exemplo, [3, p. 28-38].

\subsection{Gráfico $\Delta_{0}$ da exponencial}

Mostremos como definir o gráfico da função exponencial $x^{y}=z$ em $\mathbb{N}$ por uma fórmula $\Delta_{0}$

Para o primeiro teorema diremos que $(u, v)$ codifica os $s+1$ primeiros primos, se $u>v, r m(u, 2)=0, r m(u, c)=r m(u, p)+1$ para quaisquer dois primos consecutivos $p<c \leq v$ e, para cada primo $p \leq v, r m(u, p)=s$. Nessas condições, definimos o $i$-ésimo primo como $p(i, u, v)=$ único primo $q \leq v$ satisfazendo $r m(u, q)=i$. Dado também $w$ (produto de potências dos $s+1$ primeiros primos codificados), para cada $i \leq s$, seja $\delta(u, v, w, i)=$ a maior potência de $p(i, u, v)$ que divide $w$. É fácil escrever fórmulas $\Delta_{0}$ para cada uma dessas funções.

Teorema 2.1 (Definição $\Delta_{\mathbf{0}}$ de $\boldsymbol{x}^{\boldsymbol{y}}=\boldsymbol{z}$ ) A seguinte condição é equivalente em $\mathbb{N}$ $a x^{y}=z$ para $y>0$.

Existem $u, v, w, \eta, \xi$ tais que

(i) $(u, v)$ codifica os primeiros $s+1$ primos; 
(ii) sendo $w_{i}=\delta(u, v, w, i), \eta_{i}=r m\left(\eta, w_{i}\right)$ e $\tau_{i}=r m\left(\tau, w_{i}\right)$, temos $\eta_{0}=1, \tau_{0}=x$ $e \forall i<s\left[\left(\eta_{i+1}=2 \eta_{i} \wedge \tau_{i+1}=\tau_{i}^{2}\right) \vee\left(\eta_{i+1}=2 \eta_{i}+1 \wedge \tau_{i+1}=\tau_{i}^{2} x\right)\right]$.

(iii) $\eta_{s}=y$ e $\tau_{s}=z$.

Demonstração: Seja $\phi=\phi(x, y, z, u, v, s, w, \eta, \tau)$ a conjunção das fórmulas descritas em $i$, ii e $i i i$. Se $\phi$ for satisfatível e tomarmos $a_{i+1}=\eta_{i+1}-2 \eta_{i}$, obtemos

(A) $\eta_{i}=2^{i}+a_{1} 2^{i-1}+\cdots+a_{i}$

Caso Inicial: $i=0$.

Temos $a_{0+1}=\eta_{0+1}-2 \eta_{0}$ e $\eta_{0}=1$, e $\eta_{1}=2^{1}+a_{1}$.

Passo de Indução: $a_{i+1}=\eta_{i+1}-2 \eta_{i}$ e, por hipótese de indução, $\eta_{i}=2^{i}+a_{1} 2^{i-1}+$ $\cdots+a_{i}$, o que implica $\eta_{i+1}=2^{i+1}+a_{1} 2^{i}+\cdots+a_{i+1}$ e

(B) $\tau_{i}=x^{\eta_{i}}$.

Os dois casos a considerar $-\eta_{i+1}=2 \eta_{i} \wedge \tau_{i+1}=\tau_{i}^{2}$ e $\eta_{i+1}=2 \eta_{i}+1 \wedge \tau_{i+1}=\tau_{i}^{2} x$ — são facilmente demonstrados por indução em i.

Daí, $2^{s}+a_{1} 2^{s-1}+\cdots+a_{s-1} 2^{1}+a_{s}$ é a expansão binária de $y$ e $z=x^{y}$. E se esta é a expansão binária de $y$, podemos pegar $u, v$ que codificam os $s+1$ primeiros primos e tomamos $w, \eta, \tau$ tais que $\eta_{i}=r m\left(\eta, w_{i}\right)=2^{i}+a_{1} 2^{i-1}+\cdots+a_{i-1} 2^{1}+a_{i}$, $\tau_{i}=r m\left(\tau, w_{i}\right)=x^{\eta_{i}}$, para todo $i \leq s$, onde $u, v, s, w, \eta, \tau$ podem ser limitados por um polinômio fixo em $z$. Para $x>1$ temos $2 z^{4}$ como limitante demonstrável em $I \Delta_{0}$ na forma

$$
y>0 \wedge x>1 \wedge \exists u, v, s, w, \eta, \tau(\phi) \rightarrow \exists u, v, s, w, \eta, \tau<2 z^{4}(\phi) .
$$

A limitação de $u, v, s, w, \eta, \tau$ vem de (C) e (D) a seguir:

(C) Se $p_{j}=j$-ésimo primo, então $p_{i} \leq \tau_{i}$ e podemos escolher $w$ tal que $w_{i} \leq \tau_{i}^{2} \mathrm{e}$ $\prod_{j<i} w_{j}<\tau_{i}^{2}$, para todo $i \leq s$.

Vamos provar por indução em $i$. Para isso note que qualquer potência de $p_{i}$ que exceda $\tau_{i}$ serve como $w_{i}$; consequentemente vemos que se $p_{i} \leq \tau_{i}$, temos $w_{i} \leq \tau_{i}^{2}$.

Caso Inicial: $i=0$

Temos $\eta_{0}=1$, então $\tau_{0}=x^{\eta_{0}}=x$, $\operatorname{logo} p_{0}=2 \leq \tau_{0}$, com $\tau_{0}$ sendo uma potência de um número $\geq 2$ e teremos

$$
\prod_{j<0} w_{j}=1<\tau_{0}^{2}
$$


onde definimos o produto de uma sequência vazia como sendo 1 . zimos

Passo de indução: Assumindo a hipótese de indução e $p_{j} \mid \prod_{j<i} w_{j}, \forall j<i$, dedu-

$$
p_{i+1} \leq \prod_{j<i} w_{j}+1<\tau_{i}^{2}+1 \leq \tau_{i}^{2} \leq \tau_{i+1}
$$

Consequentemente podemos escolher $w_{i+1} \leq \tau_{i+1}^{2}$, pois $w_{i+1}=p_{i+1}^{n}$, com $n$ conveniente. E usando a hipótese de indução, $w_{i} \leq \tau_{i}^{2}$, e a definição de $\tau_{i+1}$ também temos,

$$
\prod_{j<i+1} w_{j}=\left(\prod_{j<i} w_{j}\right) w_{i}<\tau_{i}^{2} \cdot \tau_{i}^{2} \leq \tau_{i+1} \cdot \tau_{i+1}=\tau_{i+1}^{2} .
$$

(D) Se $\xi_{0}, \ldots, \xi_{j-1}$ e $\xi_{0}^{\prime}, \ldots, \xi_{j-1}^{\prime}$ são sequências definíveis por fórmulas limitadas por parâmetros então, trabalhando em $I \Delta_{0}$ e assumindo $\forall i<j\left(\xi_{i} \mid \xi\right)$ e que

$$
\forall i, k<j\left(i<k \longrightarrow m d c\left(\xi_{i}, \xi_{j}\right)=1\right) \wedge \forall i<j\left(\xi_{i}^{\prime}<\xi_{i}\right)
$$

usamos indução em k e a construção do Teorema Chinês do Resto, tomando $\xi^{*} \leq \xi \cdot k$ tal que $r m\left(\xi^{*}, \xi_{i}\right)=\xi_{i}^{\prime}$ e podemos provar

$$
k \leq j \longrightarrow \exists v \leq \xi \cdot k \forall i<k\left(r m\left(v, \xi_{i}\right)=\xi_{i}^{\prime}\right) .
$$

Aqui podemos tomar

$$
\tau^{*} \leq\left(\prod_{i<s} w_{i}\right) \cdot s \leq \tau_{s}^{2} \cdot s
$$

tal que $r m\left(\tau^{*}, w_{i}\right)=\tau_{i}$ para todo $i<s$.

Seja $\tau_{s}=z$ e como $s<z$, temos $\tau^{*} \leq z^{3}$.

E por uma aplicação adicional da construção do Teorema Chinês do Resto temos $\tau \leq \tau^{*}+\prod_{i<s+1} w_{i}$ tais que $r m\left(\tau, w_{i}\right)=\tau_{i}$ para todo $i \leq s$

$$
\tau \leq z^{3}+\left(\prod_{i<s} w_{i}\right) \cdot w_{s} \leq z^{3}+z^{2} \cdot z^{2}=z^{3}+z^{4} \leq 2 z^{4} .
$$

Portanto $\tau$ é limitado, e de forma análoga provamos a limitação das outras variáveis.

Com esta definição em mãos, podemos obter resultados interessantes. 


\subsection{Derramamento, Boa Ordem e Coleção}

A propriedade do derramamento (spillover) diz que relações $\Delta_{0} \mathrm{em} \mathbb{N}$ não limitadas devem valer em algum elemento não standard.

Teorema 2.2 (Derramamento) Seja $M \models I \Delta_{0}$ (os axiomas de $I \Delta_{0}$ são válidos em M). Então, para alguma $\varphi \in \Delta_{0}$, se $M \models \varphi(n)$ para todo $n$ standard, então existe um elemento não standard e tal que $M \models \forall x(x \leq e \longrightarrow \varphi(x))$.

Demonstração: Suponhamos que $M \models I \Delta_{0}$ e $\varphi \in \Delta_{0}$ sejam tais que $M \models \varphi(n)$ para todo $n \in \mathbb{N}$.

Se $M \models \forall x \varphi(x)$, então qualquer $e \in M \backslash \mathbb{N}$ satisfaz a conclusão do teorema.

Caso contrário, $M \models \exists x \neg \varphi$. Seja $X=\{m \in M: M \models \neg \varphi(m)\}$. Afirmamos que existe $a=\min X$ em $M$. Se não existisse tal elemento, aplicamos indução com a fórmula $\psi(x)$, dada por $\varphi(x) \wedge \forall y(y<x \longrightarrow \varphi(y))$, que é $\Delta_{0}$, pois $\varphi$ é $\Delta_{0}$. Temos que $M \models \psi(0)$ e $M \models \forall x(\psi(x) \longrightarrow \psi(x+1))$ e, portanto, $M \models \forall x \psi(x)$.

Por fim, da hipótese que $M \models \varphi(n)$ para todo $n \in \mathbb{N}$ e da existência de $a=\min X$, segue a existência do elemento $e \in M$ desejado.

Uma consequência importante é que, devido à definição $\Delta_{0}$ da exponencial, seu gráfico sempre estende-se além de $\mathbb{N}$ em qualquer modelo não standard de $I \Delta_{0}$.

Teorema 2.3 (Princípio da Boa Ordem) Seja $a \in M$ e suponha que $\unlhd$ é uma ordem parcial de a (identificado com o intervalo $\{x \in M \mid x<a\}$ ), $\unlhd \in \Delta_{0}^{M} e$ $\phi \neq X \subseteq a, X \in \Delta_{0}^{M}$. Então,

$$
\exists x \in X \forall y \in X \neg(y \unlhd x \wedge y \neq x) .
$$

Demonstração: Mostramos por indução em $i \leq \log (a)+1$ que

$$
\begin{gathered}
\exists j<2^{i}\left[\forall y \in X \exists x \in X\left(j a / 2^{i} \leq x<(j+1) a / 2^{i} \wedge x \unlhd y\right)\right] \\
\wedge \neg\left[\forall y \in X \exists x \in X\left(x<j a / 2^{i} \wedge x \unlhd y\right)\right] .
\end{gathered}
$$

Agora tomamos $i=1+\log (a)$. Então existe pelo menos um $x, \operatorname{com} j \cdot a / 2^{i} \leq x<$ $(j+1) \cdot a / 2^{i}$, e este será o elemento mínimo de $X$. 
O próximo resultado diz que segmentos iniciais de modelos de $I \Delta_{0}$ satisfazem o esquema de axiomas $B \Sigma_{1}$ (coleção para fórmulas $\Sigma_{1}$, uma fórmula é $\Sigma_{1}$ se é da forma $\left.\exists z \theta(x, y, z), \operatorname{com} \theta \in \Delta_{0}\right)$. Ele serve para introduzir uma limitação no quantificador existencial de uma fórmula $\Sigma_{1}$. Escrevemos $M \subset_{e} K$ para indicar que $M$ é segmento inicial de $K$, ou que $K$ é extensão final de $M$ (ou seja, para todo $x \in K \backslash M$ e todo $y \in M, y<x)$.

Teorema 2.4 (Axiomas $B \Sigma_{\mathbf{1}}$ ) Seja $M \subset_{e} K$ e $K \models I \Delta_{0}$. Então $M \models B \Sigma_{1}$, onde $B \Sigma_{1}$ é o $\Sigma_{1}$ esquema de coleção abaixo

$$
\forall x<y \exists z \theta(x, y, z) \longrightarrow \exists t \forall x<y \exists z<t \theta(x, y, z),
$$

para $\theta \in \Sigma_{1}$.

Demonstração: Suponha $M \models \forall x<a \exists z \theta(x, z)$, com $\theta \in \Sigma_{1}$. Sem perda de generalidade, podemos supor que $\theta$ é a fórmula $\exists \vec{u} \phi(x, z, \vec{u})$, com $\phi \in \Delta_{0}$.

Seja $M<b \in K$. Como $M \subset_{e} K, M \prec_{\Delta_{0}} K$ (ou seja, fórmulas $\Delta_{0}$ com parâmetros de $M$ e satisfeitas em $K$, também serão satisfeitas em $M$ ). Daí,

$$
K \models \forall x<a \exists z<b \exists \vec{u}<b \phi(x, y, \vec{u}) .
$$

De $K \models I \Delta_{0}$ e do Teorema 2.3, existe o menor $b$ tal que

$$
K \models \forall x<a \exists z<b \exists \vec{u}<b \phi(x, z, \vec{u}) .
$$

Seja $b^{\prime}$ o menor $b$ que satifaz $(*)$. Se $b^{\prime}>M$ então $(*)$ também valeria para $b^{\prime}-1$, pois este também excederia todo elemento de $M$, o que contradiz a escolha de $b^{\prime}$. Logo, $b^{\prime} \in M$ e como $M \prec_{\Delta_{0}} K$, temos

$$
M \models \forall x<a \exists z<b^{\prime} \exists \vec{u}<b^{\prime} \phi(x, z, \vec{u}) .
$$

E da suposição inicial vem $M \models \forall x<a \exists z<b^{\prime} \theta(x, z)$, como desejado.

Com isso, acabamos de mostrar que existe uma definição $\Delta_{0}$ para o gráfico da função exponencial e que relações $\Delta_{0}$ em $\mathbb{N}$ não limitadas devem valer em algum elemento não standard. Consequentemente, o gráfico da exponencial sempre estendese além de $\mathbb{N}$ em qualquer modelo não standard de $I \Delta_{0}$. E através do Teorema da Coleção, vimos que é possível inserir quantificador em fórmulas $\Sigma_{1}$. Estes resultados serão usados nas demonstrações do próximo capítulo. 


\section{Capítulo 3}

\section{Princípio da Casa dos Pombos e a Infinidade dos Primos}

\subsection{Introdução}

No Capítulo 1 apontamos esses dois problemas como foco desta dissertação.

Problema 1 Podemos ter $I \Delta_{0} \vdash \forall x \exists y>x(y$ primo)?

Problema 2 Podemos ter $I \Delta_{0} \vdash \Delta_{0} P H P$ ?

Lembramos que $\Delta_{0}$ PHP é o Princípio da Casa dos Pombos (do inglês, Pigeon $\boldsymbol{H}$ ole Principle) aplicado a fórmulas $\Delta_{0}$, isto é, o esquema abaixo:

$$
\forall x \leq z \exists y<z \theta(x, y) \longrightarrow \exists x_{1}, x_{2} \leq z \exists y<z\left(x_{1} \neq x_{2} \wedge \theta\left(x_{1}, y\right) \wedge \theta\left(x_{2}, y\right)\right)
$$

em que $\theta$ é fórmula $\Delta_{0}$.

Outro modo de escrevê-lo, equivalente em $I \Delta_{0}$, para $F(x, y)$ uma fórmula $\Delta_{0}$, denotamos por $\neg \exists z F: z+1 \longmapsto z$, a fórmula

$$
\left.\neg\left[\forall x<z+1 \exists ! y<z F(x, y) \wedge \forall x_{1}, x_{2} \leq z \forall y<z\left[F\left(x_{1}, y\right) \wedge F\left(x_{2}, y\right) \rightarrow x_{1}=x_{2}\right)\right]\right]
$$

isto é, não existe função injetora de $z+1$ em $z$, identificando $z \operatorname{com}\{0,1, \ldots, z-1\}$ e $z+1 \operatorname{com}\{0,1, \ldots, z\}$. 
Aqui começaremos apresentando soluções parciais para o Problema 2. A partir de $\exists x^{\log (x)}$ (veja a Definição 3.1 abaixo) para todo $x$ provaremos versões enfraquecidas de $\Delta_{0} P H P$.

Em seguida mostraremos que: se para algum elemento não standard não vale alguma forma de $\Delta_{0} P H P$, podemos encontrar uma extensão de $M$ que modela $I \Delta_{0}$ (na qual vale $I \Delta_{0}$ ) e é fechada em relação à exponenciação. E a partir destes e da conjectura que $I \Delta_{0}$ tem axiomatização finita, teremos uma versão enfraquecida de $\Delta_{0} P H P$.

Por fim demonstraremos dois teoremas (já importantes pelos métodos usados em suas provas) que serão necessários para chegarmos a uma solução parcial para o Problema 1 e veremos como este se relaciona com o Problema 2.

\subsection{O Resultado Principal}

Antes de começar a demonstrar os teoremas definiremos $\exists x^{\log (x)}$ como segue.

Definição 3.1 (A fórmula $\exists \boldsymbol{x}^{\log (x)}$ ) Primeiro definimos $[\log (x)]=y$ como sendo a fórmula $\left(2^{y} \leq x \wedge 2^{y+1}>x\right)$ que é $\Delta_{0}$, pois $x^{y}=z$ tem definição $\Delta_{0}$ (em particular $\left.2^{y}\right)$. Como $\log x<x$, para $x \geq 1$, a relação $z=x^{\log (x)}$ pode ser escrita como $\exists w<x\left(w=\log (x) \wedge z=x^{w}\right)$, que é $\Delta_{0}$ (aqui usamos novamente que $x^{y}=z$ tem definição $\left.\Delta_{0}\right)$. Por fim, a fórmula denotada por $\exists x^{\log (x)}$ é a fórmula $\exists z\left(z=x^{\log (x)}\right)$, que é $\Sigma_{1}$ (fórmula $\Delta_{0}$ com quantificador existencial não limitado).

No que segue, $\log ^{k}(x)$ denota a composição de $\log , k$ vezes (isto é, $\log ^{0}(x)=x$ e $\log ^{k+1}(x)=\log \left(\log ^{k}(x)\right)$.

O teorema abaixo mostra que de $I \Delta_{0}$ e da existência de $x^{\log ^{k}(x)}$ para todo $x$, temos uma versão enfraquecida de $\Delta_{0} P H P\left(\neg F: x^{2} \longmapsto x\right.$, com $\left.F \in \Delta_{0}\right)$. A definição de $\exists z\left(z=x^{\log ^{k}(x)}\right)$ segue as mesmas ideias da definição acima.

Teorema 3.1 Para $k \in N$ e $F \in \Delta_{0}$,

$$
I \Delta_{0} \vdash \forall x\left(\exists x^{\log ^{k}(x)} \wedge x>1 \longrightarrow \neg F: x^{2} \longmapsto x\right) .
$$


Demonstração: Vamos começar com o caso $k=1$. Para isso assumimos que para algum $a \in M, a>1, M \models \exists a^{\log (a)}$ e $F: a^{2} \longmapsto a \operatorname{com} F \in \Delta_{0}^{M}$. Suponha que $a^{b} \in M$ e seja $G: 2^{b} \longrightarrow a \operatorname{com} G \in \Delta_{0}^{M}$. Pense em $G$ como uma árvore binária de altura b com nó $\sigma$ associado a $G(\sigma)$, por exemplo, para $b=3$, associamos a sequência 00 com $F(G(000)+a \cdot G(001))<a, 01$ com $F(G(010)+a \cdot G(011))<a, 0$ com $F(F(G(000)+a \cdot G(001))+a \cdot F(G(010)+a \cdot G(011)))<a$ e assim por diante até finalmente associarmos $\phi$ com algum valor $c<a$. Como $F$ é injetora, $G$ determina $c$ de modo único e vice versa.

Fixo $b$ tal que $a^{b} \in M$, e para $c<a$ e $d \leq b$ definimos $H_{c}^{d}: \subseteq 2^{d} \longrightarrow a$ como $H_{c}^{d}(\sigma)=e$ se, e somente se, existir sequência $e_{0}, e_{1}, \ldots, e_{d}<a$, tal que

$$
\begin{aligned}
e_{0}=c \wedge e_{d}= & e \wedge \forall 0<i \leq d\left[\sigma(i-1)=0 \longrightarrow \exists x<a F\left(e_{i}+a \cdot x\right)=e_{i-1}\right] \\
& \wedge\left[\sigma(i-1)=1 \longrightarrow \exists x<a F\left(x+a \cdot e_{i}\right)=e_{i-1}\right]
\end{aligned}
$$

onde identificamos $\sigma<2^{d}$ com a função $\sigma: d \longmapsto 2$ que leva $x<d$ em zero se, e somente se, o coeficiente de $2^{x}$ na expansão binária de $\sigma$ é zero.

Como o gráfico da exponenciação é $\Delta_{0}$ (ver Teorema 2.1) a sequência $e_{0}, e_{1}, \ldots, e_{d}$ pode ser codificada por um número na base $a$ de tamanho menor que $a^{d+1} \leq a^{b+1}$, usando indução em $d \leq b$, na fórmula que exprime $e_{0}+a \cdot e_{1}+\cdots+a^{d} \cdot e_{d} \leq a^{d+1} \leq a^{b+1}$.

Da hipótese de indução e porque $e_{i}<a$, para todo $0 \leq i \leq d$, temos,

$$
e_{0}+a \cdot e_{1}+\cdots+a^{d} \cdot e_{d} \leq a^{d}+a^{d} \cdot e_{d} \leq a^{d}\left(1+e_{d}\right) \leq a^{d+1} \leq a^{b+1} .
$$

Afirmação: Se $G \in \Delta_{0}$ e $G: 2^{b} \longrightarrow a$ então $\exists c<a\left(H_{c}^{b}=G\right)$.

Demonstração: Vamos provar por indução em $d \leq b$ que

$$
\forall \sigma<2^{b-d} \exists c<a \forall \tau<2^{d} H_{c}^{d}(\tau)=G(\sigma * \tau)
$$

onde $\sigma * \tau$ é o código da função $\sigma * \tau: b \longrightarrow 2$ dada por $\sigma * \tau(x)=\sigma(x), 0<x<b-d$ e $\sigma * \tau(x)=\tau(x-b+d), b-d \leq x<b$ é a concatenação de $\sigma$ e $\tau$. Como a fórmula é $\Delta_{0}$, podemos provar a indução usando $I \Delta_{0}$.

Por hipótese de indução temos, $H_{c_{0}}^{d-1}(\tau)=G(\sigma * 0 * \tau), H_{c_{1}}^{d-1}(\tau)=G(\sigma * 1 * \tau)$, para $\sigma<2^{b-d}$ e $\tau<2^{d-1}$.

Então, para $\tau<2^{d}$, temos dois casos

(i) Se $\tau$ começa com 0 temos $G(\sigma * \tau)=G\left(\sigma * 0 * \tau^{\prime}\right)=H_{c_{0}}^{d-1}\left(\tau^{\prime}\right)$. 
(ii) Se $\tau$ começa com 1 temos $G(\sigma * \tau)=G\left(\sigma * 1 * \tau^{\prime}\right)=H_{c_{1}}^{d-1}\left(\tau^{\prime}\right)$.

Daí, $H_{c}^{d}(\tau)=G(\sigma * \tau)$ para $\tau<2^{d}$, onde $c=F\left(c_{0}+a \cdot c_{1}\right)$. Isso demonstra a afirmação.

Agora fica fácil obter a contradição desejada. Para isso tomo $b=1+\log (a)$ e vemos que $c<a \longmapsto H_{c}^{b}$ nos dá uma função sobrejetora de $a$ em todos os mapas $\Delta_{0}$ de $2^{1+\log (a)}$ em $a$. Daí, a função $G$ que leva $x<2^{1+\log (a)}$ em $1 \dot{-} H_{\min (x, a-1)}^{b}(a \dot{-} b=a-b$, se $a \geq b$ e $a \dot{-} b=a-b$, se $a<b)$ está em $\Delta_{0}^{M}$ e para $\forall c<a$ temos:

- Se $H_{c}^{b}(c)=0$, temos $1 \dot{-} H_{\min (c, a-1)}^{b}(c)=1 \dot{-} H_{c}^{b}(c)=1$.

- E se $H_{c}^{b}(c)=1$, temos $1 \dot{-} H_{\min (c, a-1)}^{b}(c)=0$.

Portanto $G$ está em $\Delta_{0}^{M}$, mas não é igual a nenhuma $H_{c}^{b}$ para nenhum $c<a$ e temos a contradição procurada, provando o caso $k=1$.

Agora suponhamos $k=2$, isto é, $M=\exists a^{\log ^{2}(a)}$. Então podemos novamente provar a afirmação para $b=2+\log ^{2}(a)$. Isso significa que temos uma função $H^{b} \in \Delta_{0}^{M}$ tal que para $c<a$, as funções $H_{c}^{b}$ enumeram todos os mapas $\Delta_{0}^{M}$ de $2^{2+\log ^{2}(a)}$ em $a$, em particular de $\log (a)+1\left(\leq 2^{2+\log ^{2}(a)}\right)$ em $a$.

Obs: Como $2^{2+\log ^{2}(a)}=2^{2} \cdot 2^{\log (\log (a))} \leq 4 \cdot \log (a)<a$, a diagonalização do caso $k=1$ não funciona, pois para $c>4 \cdot \log (a)$ não podemos calcular $H_{c}^{b}(c)$.

Da restrição acima, podemos usar $H_{c}^{b}$ como enumeração das $G: 1+\log (a) \subseteq$ $2^{2+\log ^{2}(a)} \longrightarrow a$. Agora construímos $\tilde{H}_{c}^{b}$, com $\tilde{b} \leq 1+\log (a)$ como no caso $k=1$, mas usando $H_{c}^{b}$ para codificar $e_{0}, e_{1}, \ldots, e_{d}$ (existe $c<a$ tal que $H_{c}^{b}(0)=e_{0}, H_{c}^{b}(d)=e_{d}$ e $\left.\forall \sigma<j<d H_{c}^{b}(j)=e_{j} \wedge \ldots\right)$. Então, $\tilde{H}_{\tilde{c}}^{1+\log (a)}$ codifica todos os mapas $\Delta_{0}$ de $1+\log (a)$ em $a$ e obtemos de modo similar a contradição desejada.

Repetindo o processo duas vezes provamos o caso $k=3$ e assim por diante.

Do teorema anterior temos, o corolário a seguir, que mostra uma versão mais forte de $\Delta_{0} P H P(\neg \exists x F:(1+\epsilon) x \longmapsto x$, com $(1+\epsilon) x>x)$ a partir de $\exists x^{\log (x)}$, para todo $x$.

Corolário 3.1 Para $F \in \Delta_{0} e \epsilon>0$ racional, temos

$$
I \Delta_{0}+\forall x\left(\exists x^{\log x}\right) \vdash \neg \exists x F:(1+\epsilon) x \longmapsto x \wedge(1+\epsilon) x>x .
$$


Demonstração: Suponhamos que $\forall x\left(\exists x^{\log x}\right)$ vale em M e que existe $F \in \Delta_{0}^{M}$, $\operatorname{com} F:(1+\epsilon) a \longmapsto a$ e $(1+\epsilon) a>a, a \in M$.

Então $a$ tem que ser não standard e claramente $F$ nos dará um mapa $\Delta_{0}^{M}$ de $(1+\epsilon) a+\epsilon \cdot a \longmapsto(1+\epsilon) a$, e assim por diante. Assim podemos assumir, sem perda de generalidade $\epsilon=3$.

Agora tomamos $G \in \Delta_{0}^{M}, G: a^{2} \longmapsto a^{2}$ dada por $G(4 a b+c)=a b+F(c)$, com $0 \leq c<4 a$.

Então para $4 a \leq x<a^{2}$ e usando que $F(c) \leq a, x=4 a b+c$ e $b \geq 1$, temos

$$
G(x) \leq a b+a \leq a b+a b=2 a b \leq 2 a b+\frac{c}{2}=\frac{x}{2},
$$

ou seja, $G(x) \leq \frac{x}{2}$.

E para $x<4 a$, temos $x=c$ e da definição de $\mathrm{G}$ vem

$$
G(x)=G(c)=F(c)<a .
$$

Consequentemente $G^{1+\log (a)}: a^{2} \longmapsto \max \left(4 a, a^{2} / 2^{1+\log (a)}\right) \leq 4 a$.

Daí, $G^{2+\log (a)}: a^{2} \longmapsto a$, o que contraria o Teorema 3.1, pois $\left(a^{2}\right)^{2+\log (a)} \in M \mathrm{e}$ $G^{2+\log (a)}$ é definível em $\Delta_{0}^{M}$.

Observação: A condição $\forall x\left(\exists x^{\log x}\right)$ pode ser enfraquecida, ver A. Atserias, [1].

\subsection{Variantes}

O próximo Teorema terá papel fundamental na demonstração do Teorema 3.3. Ele nos diz que: se para algum elemento não standard $a$ não vale alguma forma de $\Delta_{0} P H P$ $\left(F: a^{\gamma} \longmapsto a\right.$ ), podemos encontrar uma extensão final $K$ de $M$ (ver definição no Teorema 2.4) que modela $I \Delta_{0}$.

Teorema 3.2 Suponha $a^{\gamma} \in M, F \in \Delta_{0}^{M}$ e $F: a^{\gamma} \longmapsto a$. Então existe uma extensão final $K$ de $M\left(M \subseteq_{e} K\right)$ tal que $K \models I \Delta_{0}$ e e $a^{\gamma^{2}} \in K$.

Demonstração: Podemos assumir $a^{\gamma^{2}} \in M$, pois caso contrário estaria em $K$ e nada teríamos para provar. 
Assim como no Teorema 3.1 podemos encontrar uma função $H \in \Delta_{0}^{M}$ tal que $\left\{H_{c} \mid c<a\right\}$ enumera todos os mapas $\Delta_{0}^{M}$ de $2^{\gamma}$ em $a$. A ideia agora é tratar $c<a$ como o "número" $\Sigma_{i<2^{\gamma}} H_{c}(i) \cdot a^{i}$.

Agora para $q, e, c, d<a$ e $j<2^{\gamma}$ definimos as seguintes funções.

1. $c \oplus d=e$ (a soma de $c$ com $d$ é $e$ ) como sendo a fórmula

$$
\begin{gathered}
\exists y<a \forall i<2^{\gamma}\left[H_{y}(0)=0 \wedge\left(i+1<2^{\gamma} \longrightarrow\right.\right. \\
\left.H_{y}(i)+H_{c}(i)+H_{d}(i)=H_{e}(i)+a \cdot H_{y}(i+1)\right) \\
\left.\wedge\left(i+1=2^{\gamma} \longrightarrow H_{y}(i)+H_{c}(i)+H_{d}(i)=H_{e}(i)\right)\right] .
\end{gathered}
$$

2. $q_{j}^{*} c=e\left(\right.$ a soma de $q \cdot a^{j}$ cópias de c é $\left.e\right)$ :

$$
\begin{gathered}
\exists y<a \forall i<2^{\gamma}\left[\left(i<j \longrightarrow H_{y}(i)=H_{e}(i)=0\right)\right. \\
\wedge\left(j<i+1<2^{\gamma} \longrightarrow H_{y}(i)+q \cdot H_{c}(i-j)=H_{e}(i)+a \cdot H_{y}(i+1)\right) \\
\wedge\left(j<i+1=2^{\gamma} \longrightarrow H_{y}(i)+q \cdot H_{c}(i-j)=H_{e}(i)\right) \\
\left.\wedge\left(2^{\gamma}-j<i<2^{\gamma} \longrightarrow H_{c}(i)=0 \vee q=0\right)\right] .
\end{gathered}
$$

3. $c \otimes d=e($ a multiplicação de $c \operatorname{com} d$ é $e)$ :

$$
\begin{aligned}
& \exists y<a \forall i<2^{\gamma}\left[H_{y}(0)=H_{c}(0)_{0}^{*} d \wedge e=H_{y}\left(2^{\gamma}-1\right)\right. \\
& \left.\wedge\left(i+1<2^{\gamma} \longrightarrow H_{y}(i+1)=H_{y}(i) \oplus\left(H_{c}(i)_{i}^{*} d\right)\right)\right]
\end{aligned}
$$

Agora sejam $I \subseteq_{e} 2^{\gamma}$, com $\gamma^{2} \in I$ e $I$ fechado pela adição e

$$
K=\left\{c<a \mid \exists t \in I \forall t<i<2^{\gamma} H_{c}(i)=0\right\} .
$$

Então $K$ é fechado sobre $\oplus, \otimes$ e para $c, d<a$ satisfazem

$$
c \unlhd d \in K \longrightarrow c \in K,
$$

onde

$$
c \unlhd d \longleftrightarrow \exists e<a(c \oplus e=d) .
$$

Consequentemente podemos tratar $K$ como uma estrutura da linguagem aritmética, com menor que, restringindo $\oplus, \otimes, \unlhd$ para $K$ e identificando $c, d \in K$ se $H_{c}=H_{d}$. 
Então $K$ satisfaz os axiomas básicos da aritmética, com excessão da indução, e como $\otimes, \oplus, \unlhd \in \Delta_{0}^{M}$ temos que se $A \subseteq K$ esta em $\Delta_{0}^{k}$ então $A=K \cap B$ para algum $B \subseteq a, B \in \Delta_{0}^{M}$. Aqui usamos o Teorema 2.3 para concluir que $K \models I \Delta_{0}$.

Também via isomorfismo, $M \subset_{e} K$. Para ver isso, seja $q \in M, q=\sum_{j=0}^{m} q_{j} \cdot a^{j}$ na base $a$. Como $a^{\gamma^{2}} \notin M$ temos $m<\gamma^{2}$ então, $m \in I$, pois $\gamma^{2} \in I$, e existe $c<a$ tal que

$$
H_{c}(i)=\left\{\begin{array}{rr}
q_{i} & \text { para } i<m, \\
0 & \text { para } m \leq i<2^{\gamma} .
\end{array}\right.
$$

Logo, identificando $q$ com $c$ temos a imersão de $M$ como segmento inicial de $K$ (ver a definição de $K$ ).

Só resta mostrar que $a^{\gamma^{2}} \in K$. Como $K$ é uma extensão própria de $M$ e as hipóteses do teorema continuam valendo para $K$, então se $a^{\gamma^{2}} \notin K$, podemos repetir a construção para ter $K^{(2)}, \ldots$ Se repetirmos $\omega_{1}$ vezes teremos $K^{\left(\omega_{1}\right)}$, uma extensão final de tipo $\omega_{1}$ de $M$. Logo, $K^{\left(\omega_{1}\right)} \models I \Delta_{0}+$ esquema coleção; daí por resultados em [6], $K^{\left(\omega_{1}\right)} \models$ Axiomas de Peano e $a^{\gamma^{2}} \in K^{\left(\omega_{1}\right)}$, tendo $a^{\gamma^{2}} \in K^{(\alpha)}$ para algum $\alpha<\omega_{1}$.

Fica claro que com um pouco mais de esforço podemos mostrar que $a^{2^{\gamma}} \in K$ e repetindo o processo teremos o corolário abaixo. Que nos dá uma extensão final fechada em relação exponenciação.

Corolário 3.2 Suponha que $a^{\gamma} \in M, F \in \Delta_{0}^{M}$ e $F: a^{\gamma} \longmapsto a$. Então existe uma extensão final $K$ de $M$ tal que $K \models I \Delta_{0} e\left\{\delta \mid a^{\delta} \in K\right\}$ é fechado pela exponenciação.

Aqui vemos que se existir uma axiomatização fintita de $I \Delta_{0}$, então vale uma forma enfraquecida de $\Delta_{0} P H P\left(\neg \exists x>1, F: x^{2} \longmapsto x\right)$.

Teorema 3.3 Se I $\Delta_{0}$ for finitamente axiomatizável e $F \in \Delta_{0}$, então

$$
I \Delta_{0} \vdash \neg \exists x>1, F: x^{2} \longmapsto x .
$$

Demonstração: Suponhamos que $I \Delta_{0}$ seja finitamente axiomatizável, $M_{0} \models$ $I \Delta_{0}, 1<a \in M_{0}, F \in \Delta_{0}$ e $F: a^{2} \longmapsto a$ e vamos chegar a uma contradição.

Tomando uma potência tão grande quanto necessária podemos assumir $a^{\gamma} \in M_{0}$, para algum termo não standard $a$ e que $\gamma=2^{\lambda}$, para algum $\lambda$. 
Também podemos assumir que o único parâmetro $c$ aparecendo na definição $\Delta_{0}$ de $F$ é menor que $a^{\gamma}$.

Sendo $M$ a subestrutura de $M_{0}$ com domínio $\left\{x \in M_{0} \mid x<a^{n \cdot \gamma}, n \in N\right\}$.

Daí, $M \models I \Delta_{0}\left(M_{0} \models I \Delta_{0}\right), F \in \Delta_{0}^{M}$ e $F: a^{2} \longmapsto a$ em $M$.

Agora defino em $M$ a função $G: a^{\gamma} \longmapsto a^{\gamma}$ como segue.

Dado $b<a^{\gamma}$, seja $b=\Sigma_{i<2^{\lambda-1}} b_{i} \cdot a^{2 i}$ na base $a^{2}$, e seja

$$
G(b)=\Sigma_{i<2^{\lambda-1}} F\left(b_{i}\right) \cdot a^{i} .
$$

Como $F: a^{2} \longmapsto a$, temos

$G(b) \leq \Sigma_{i<2^{\lambda-1}}(a-1) \cdot a^{i}=\Sigma_{i<2^{\lambda-1}}\left(a^{i+1}-a^{i}\right)=a^{\left(2^{\lambda-1}-1\right)+1}-a^{0}=a^{2^{\lambda-1}}-1$.

E, como $\gamma=2^{\lambda}$, temos $\frac{\gamma}{2}=2^{\lambda-1} \mathrm{e}$

$$
G(b) \leq a^{\frac{\gamma}{2}}-1<a^{\frac{\gamma}{2}} .
$$

Seja $H=G^{\lambda}$ e lembrando que $\gamma=2^{\lambda}$ e $G: a^{\gamma} \longmapsto a^{\gamma}$, então $H: a^{\gamma} \longmapsto a$. E como $G(b)<a^{\gamma / 2}$. Na base $a^{2}, G(b)=\sum_{i<2^{\lambda-2}} F\left(G(b)_{i}\right) a^{2 i}$. Daí, $G^{2}(b)=G(G(b))<a^{\gamma / 4}$, etc. No fim, para $b<a^{\gamma}$, a sequência $b, G(b), \ldots, G^{\lambda}(b)$ pode ser codificada pelo elemento

$b+G(b) a^{\gamma}+G^{2}(b) a^{\gamma+\gamma / 2}+G^{3}(b) a^{\gamma+\gamma / 2+\gamma / 4}+\cdots+G^{\lambda}(b) a^{\gamma+\gamma / 2+\gamma / 4+\cdots}<a^{\gamma+\gamma}=a^{2 \gamma}$

e temos que $H \in \Delta_{0}^{M}$ (com parâmetros $a, a^{\gamma}, \gamma, c$ ).

Seja $\left\{\theta_{0}, \theta_{1}, \ldots, \theta_{k-3}\right\}$ uma axiomatização finita de $I \Delta_{0}$, e também temos $H$ : $a^{\gamma} \longmapsto a, \neg \exists x\left(x=a^{\gamma^{2}}\right)$ na forma prenex nomal abaixo

$$
\forall y_{0} \exists x_{1}<t_{1} \forall y_{1}<s_{1} \exists x_{2}<t_{2} \forall y_{2}<s_{2} \ldots \exists x_{m}<t_{m} \forall y_{m}<s_{m} \psi_{j}, j<k,
$$

onde $\psi_{j}=\psi_{j}\left(\vec{x}, \vec{y}, a, \gamma, c, a^{\gamma}\right)$ é livre de quantificadores e

$$
\begin{gathered}
t_{i}=t_{i}\left(x_{1}, \ldots, x_{i-1}, y_{1}, \ldots, y_{i-1}, a, \gamma, c, a^{\gamma}\right), \\
s_{i}=s_{i}\left(x_{1}, \ldots, x_{i}, y_{1}, \ldots, y_{i}, a, \gamma, c, a^{\gamma}\right)
\end{gathered}
$$

são termos. 
Agora definimos a função $\Omega$ em $N$ como segue. Para $q \in N$, se $q=\left\langle 0,\left\langle j, e, u_{1}\right.\right.$, $\left.\left.\ldots, u_{m}, w_{0}, \ldots, w_{m}\right\rangle\right\rangle$ com $j \leq k$ e $1 \leq e \leq m$, seja $\Omega(q)$ o menor $x_{e}$ tal que $x_{e}<t_{e}^{\prime}$ e $\forall y_{e}<s_{e}^{\prime} \exists x_{e+1}<t_{e+1}^{\prime} \ldots \exists x_{m}<t_{m}^{\prime} \forall y_{m}<s_{m}^{\prime} \psi_{j}^{\prime}$ onde $\psi_{j}^{\prime}=\psi_{j}\left(\Omega\left(u_{1}\right)\right.$, $\left.\ldots, \Omega\left(u_{e-1}\right), \ldots, x_{e}, \ldots, x_{m}, \Omega\left(w_{0}\right), \ldots, \Omega\left(w_{e-1}\right), y_{e}, \ldots, y_{m}, a, \gamma, c, a^{\gamma}\right), t_{i}^{\prime}=$ $t_{i}\left(\Omega\left(u_{1}\right), \ldots, \Omega\left(u_{e-1}\right), \ldots, x_{e}, \ldots, x_{i-1}, \Omega\left(w_{0}\right), \ldots, \Omega\left(w_{e-1}\right), y_{e}, \ldots, y_{i-1}, a, \gamma, c, a^{\gamma}\right), \mathrm{e}$ $s_{i}^{\prime}=s_{i}\left(\Omega\left(u_{1}\right), \ldots, \Omega\left(u_{e-1}\right), \ldots, x_{e}, \ldots, x_{i}, \Omega\left(w_{0}\right), \ldots, \Omega\left(w_{e-1}\right), y_{e}, \ldots, y_{i-1}, a, \gamma, c, a^{\gamma}\right)$, se existir tal $x_{e}$, e $s_{i}^{\prime}=0$ caso contrário. Se

- $q=\langle 1, u\rangle$ temos $\Omega(q)=0$,

- $q=\langle 2, u\rangle$ temos $\Omega(q)=1$,

- $q=\langle 3, u\rangle$ temos $\Omega(q)=a$,

- $q=\langle 4, u\rangle$ temos $\Omega(q)=\gamma$,

- $q=\langle 5, u\rangle$ temos $\Omega(q)=c$,

- $q=\langle 6, u\rangle$ temos $\Omega(q)=a^{\gamma}$,

- $q=\left\langle 7,\left\langle u_{1}, u_{2}\right\rangle\right\rangle$ temos $\Omega(q)=\Omega\left(u_{1}\right) \cdot \Omega\left(u_{2}\right)$,

- $q=\left\langle 8,\left\langle u_{1}, u_{2}\right\rangle\right\rangle$ temos $\Omega(q)=\Omega\left(u_{1}\right)+\Omega\left(u_{2}\right)$,

- caso contrário, $\Omega(q)=0$.

Seja $J=\Omega^{\prime \prime} N(J$ é a imagem de $N$ por $\Omega$ ). Então $J$ é uma subestrutura de $M$ e uma subestrutura elementar para todas as subfórmulas de

$$
\theta_{0}, \theta_{1}, \ldots, \theta_{k-3}, H: a^{\gamma} \longmapsto a, \neg \exists x\left(x=a^{\gamma^{2}}\right) .
$$

Consequentemente, $J=I \Delta_{0}+H: a^{\gamma} \longmapsto a+\neg \exists x\left(x=a^{\gamma^{2}}\right)+a^{\gamma}$ e, do Teorema 3.2, $J$ tem uma extensão final própria de um modelo de $I \Delta_{0}$. E do Teorema 2.4, temos $J \models B \Sigma_{1}$.

Agora vamos obter uma contradição mostrando que a conclusão imediatamente anterior é falsa. Note que existe uma fórmula $\Sigma_{1}$ que expressa $\Omega(n)=x, \forall n \in N$, pois $\Omega$ é definida por recursão, em que usamos a função $\boldsymbol{\beta}$ de Gödel para codificar essa sequência e o restante é decodificação de sequências codificadas por $q$, o que é $\Delta_{0}$ (cada elemento dessa seq. é menor que " $q$ "). E ainda mais, temos que $J \models \Omega(n)=x$ 
para todos $n, x \in J$ tais que $M \models \Omega(n)=x$; também, $J \models b=a^{n \gamma}$ se $M \models b=a^{n \gamma}$ (pois $J$ é subestrutura e $a^{\gamma} \in J$ ); por fim, $J$ é a imagem de $N$ por $\Omega$. Consequetemente

$$
J \models \forall x<a \exists n \exists b\left(b=a^{n \cdot \gamma} \wedge \Omega(n)=x\right) .
$$

E como existem infinitos $x \in J$ menores que $a$, então $b$ não pode ser limitado por nenhum elemento de $J$, pois $\left\{a^{n \cdot \gamma} \mid n \in N\right\}$ é cofinal em $M$ e consequentemente em $J$ (Como $a^{\gamma}$ é o mesmo em $J$ e $M$, garante que os $a^{n \cdot \gamma}$ também sejam os mesmos). Logo $B \Sigma_{1}$ não vale em $J$ e temos o desejado.

Os especialistas na área acreditam que a hipótese de $I \Delta_{0}$ ser finitamente axiomatizável seja falsa. Veja os comentários ao Problema Aberto 3 na página 30.

\subsection{Primos e $\mathbf{P H P}$}

O Teorema 3.6 na página 25, que será demonstrado mais adiante, diz que se não existirem primos entre $a \in M$ (não standard) e $a^{11}$, então uma forma fraca do $P H P$ não vale em $M$, ou seja, existe $F \in \Delta_{0}^{M}, F: 9 a \cdot \log (a) \longmapsto 8 a \cdot \log (a)$.

Para prová-lo precisaremos de dois resultados em codificação, que são de interesse independente. E através deles mostraremos que podemos definir, em $\Delta_{0}^{M}$, uma função que é uma somatória de tamanho variável $\left(\sum_{i \leq j} G_{t}(i)\right)$.

Teorema 3.4 Seja $a \in M, d \leq \log (a)^{k}$, e $b<2^{\log (a)^{\alpha}}$, com $\alpha$ racional standard, e $0<\alpha<1$. Seja $G \in \Delta_{0}^{M}, G: M^{2} \longrightarrow M$. Então, existe uma função $F \in \Delta_{0}^{M}$ (uniforme) tal que $F(0)=b$ e para $i+1 \leq d$

$$
F(i+1)=\min \left(G(F(i), i), 2^{\log (a)^{\alpha}}\right) .
$$

Obs: $\log (a)^{k}$ é a potência de base $\log (a)$ e expoente $k$.

Demonstração: Podemos assumir que $a$ é muito grande e $G: M^{2} \longrightarrow 2^{\log (a)^{\alpha}}$.

Primeiro vamos provar o resultado para toda $G$ substituindo $k$ por $(1-\alpha) / 2$. Nesse caso a definição, para $i \leq \log (a)^{(1-\alpha) / 2}$, de $F(i)$ é

$$
F(i)=z \text { se, e somente se } \exists \text { sequência } w_{0}, w_{1}, \ldots, w_{i} \text { tais que } w_{0}=b \wedge w_{i}=z
$$

$$
\wedge \forall 0 \leq j<i\left(w_{j+1}=G\left(w_{j}, j\right)\right) .
$$


O que nos dá $F \in \Delta_{0}^{M}$, pois a sequência $w_{0}, w_{1}, \ldots, w_{i}$ pode ser codificada na base $2^{\log (a)^{\alpha}}$ (pois $\left.b, G\left(w_{j}, j\right)<2^{\log (a)^{\alpha}}, \forall j<i\right)$, por $i+\sum_{j=1}^{i+1} w_{j-1} B^{j}$, onde $B=2^{\log (a)^{\alpha}}$ é a base. Como $i \leq \log (a)^{(1-\alpha) / 2}$, temos

$$
i+\sum_{j=1}^{i+1} w_{j-1} B^{j}=B^{i+2} \leq B^{2+\log (a)^{(1-\alpha) / 2}}=\left(2^{\log (a)^{\alpha}}\right)^{2+\log (a)^{(1-\alpha) / 2}} .
$$

Daí,

$$
\left(2^{\log (a)^{\alpha}}\right)^{2+\log (a)^{(1-\alpha) / 2}}=2^{2 \log (a)^{\alpha}+\log (a)^{\alpha} \cdot \log (a)^{(1-\alpha) / 2}}=2^{2 \log (a)^{\alpha}+\log (a)^{(1+\alpha) / 2}}
$$

e como $0<\alpha<1$ temos, $\alpha=\frac{\alpha+\alpha}{2}<\frac{1+\alpha}{2}$ e

$$
\left(2^{\log (a)^{\alpha}}\right)^{2+\log (a)^{(1-\alpha) / 2}} \leq 2^{3 \log (a)^{(1+\alpha) / 2}}
$$

e $0<\alpha<1$ equivale a $1 / 2<(1+\alpha) / 2<1$, então

$$
\left(2^{\log (a)^{\alpha}}\right)^{2+\log (a)^{(1-\alpha) / 2}} \leq 2^{3 \log (a)^{(1+\alpha) / 2}}<2^{\log (a)} \leq a .
$$

Agora assumimos o resultado, para todas as tais $G$, substituindo $k$ por $\beta$. Vamos ver como trocar $\beta$ por $\beta+(1-\alpha) / 2$ e isso nos dará a demonstração do teorema desejado.

Então para tais $G$, tomamos $G_{y, w}$ como

$$
G_{y, w}(x, u)=G(x, y+u)
$$

e $F_{y, w}$ a função $\Delta_{0}^{M}$, uniforme em $y$ e $w, \operatorname{com} u<\log (a)^{\beta}$,

$$
F_{y, w}(0)=w, F_{y, w}(u+1)=G_{y, w}\left(F_{y, w}(u), u\right)
$$

Então, para $i \leq \log (a)^{\beta} \cdot \log (a)^{(1-\alpha) / 2}, F(i)$ é dada por

$$
\begin{gathered}
F(i)=z \text { se, e somente se, } \exists \text { sequência } y_{0}, y_{1}, \ldots, y_{j}, w_{0}, w_{1}, \ldots, w_{j} \text { tal que } \\
y_{0}=0 \wedge y_{j}=i \wedge \forall 0 \leq t<j\left(0 \leq y_{t+1}-y_{t} \leq \log (a)^{\beta}\right) \wedge j \leq \log (a)^{(1-\alpha) / 2} \\
\wedge w_{0}=b \wedge w_{j}=z \wedge \forall 0 \leq t<j, F_{y_{t}, w_{t}}\left(y_{t+1}\right)=w_{t+1}
\end{gathered}
$$

Essa definição mostra que $F \in \Delta_{0}^{M}$, pois a sequência $y_{0}, y_{1}, \ldots, y_{j}$ pode ser codificada por um número $\leq\left(1-\log (a)^{\beta+(1-\alpha) / 2}\right)^{1+\log (a)^{(1-\alpha) / 2}}<a$ e a sequência $w_{0}, w_{1}, \ldots, w_{j}$ pode ser codificada por um número $\leq\left(1+2^{\log (a)^{\alpha}}\right)^{2+\log (a)^{(1-\alpha) / 2}}<a$.

O segundo resultado requerido é o seguinte. 
Teorema 3.5 Sejam $a, b \in M, d \leq \log (a)^{k}$ e $G \in \Delta_{0}^{M}, G: b \longrightarrow b$. Então existe uma função $F \in \Delta_{0}^{M}$ uniforme tal que $F(0)=G(0)$ e para todo $i<d$,

$$
F(i+1)=F(i)+G(i+1) .
$$

Obs: $[x]=$ parte inteira de $x$.

Demonstração: Daqui em diante usaremos a notação $\sum_{q \leq i} G(q)$ para $F(i)$.

Note que o resultado é verdadeiro se $b \leq d$, pelo Teorema 3.4.

Seja $G(i)=\sum_{s \leq \log (b)} G_{s}(i) \cdot 2^{s}$ em notação binária. Agora definiremos as funções $p_{s}, H_{t} \in \Delta_{0}^{M}$ tais que para $0 \leq j \leq d$ e $t \leq \log (b)$

$$
\sum_{i \leq j}\left(\sum_{s \leq t} G_{s}(i) \cdot 2^{s}\right)=\sum_{s<t} p_{s} \cdot 2^{s}+2^{t} \cdot H_{t}(j)
$$

com $p_{s}(j) \in\{0,1\}$. Para isso, com $0 \leq j \leq d$, definimos

$$
H_{0}(j)=\sum_{i \leq j} G_{0}(i)
$$

e

$$
H_{t+1}(j+1)=\min \left(\left[\frac{H_{t}(j)}{2}\right]+\sum_{i \leq j} G_{t+1}(i),(t+1) \cdot d\right)=\left[\frac{H_{t}(j)}{2}\right]+\sum_{i \leq j} G_{t+1}(i)
$$

pois, $\Sigma_{i \leq j} G_{t}(i) \leq j \leq d$ (cada $G_{t}(i)=0$ ou 1 ) e prova-se por indução em $t$ que $\left[\frac{H_{t}(j)}{2}\right] \leq t \cdot j \leq t \cdot d$.

Então $H \in \Delta_{0}^{M}$ pelo teorema anterior.

Agora para $j \leq d$ e $t \leq \log (b)$, definimos

$$
p_{t}(j)= \begin{cases}0 & \text { se } H_{t}(j) \text { é par, } \\ 1 & \text { se } H_{t}(j) \text { é ímpar. }\end{cases}
$$

Então, por indução em $t$ na fórmula que define $H$ e usando que $G_{t}(0)=1$ ou 0 , temos $p_{t}(0)=G_{t}(0)$. E para $0 \leq j<d$ vamos mostrar por indução em $t<\log (b)$ que

$$
2^{t} \cdot H_{t}(j)+\sum_{s<t} p_{s}(j) \cdot 2^{s}+\sum_{s \leq t} G_{s}(j+1) \cdot 2^{s}=2^{t} \cdot H_{t}(j+1)+\sum_{s<t} p_{s}(j+1) \cdot 2^{s}
$$


onde $F(j)=2^{t} \cdot H_{t}(j)+\sum_{s<t} p_{s}(j) \cdot 2^{s}$ e $G(j)=\sum_{s \leq t} G_{s}(j+1) \cdot 2^{s}$.

Caso Inicial: Usando duas vezes a definição de $H_{0}$ temos

$$
\begin{gathered}
H_{0}(j+1)=\sum_{i \leq j+1} G_{0}(i) \\
H_{0}(j+1)=H_{0}(j)+G_{0}(j+1) .
\end{gathered}
$$

Passo de Indução:

$$
\begin{gathered}
2^{t+1} \cdot H_{t+1}(j)+\sum_{s<t+1} p_{s}(j) \cdot 2^{s}+\sum_{s \leq t+1} G_{s}(j+1) \cdot 2^{s}= \\
2^{t+1} \cdot\left(\left[\frac{H_{t}(j)}{2}\right]+\sum_{i \leq j} G_{t+1}(i)\right)+\sum_{s<t} p_{s}(j) \cdot 2^{s}+\sum_{s \leq t} G_{s}(j+1) \cdot 2^{s}+G_{t+1}(j+1) \cdot 2^{t+1}+p_{t}(j) \cdot 2^{t}
\end{gathered}
$$

Temos dois casos a considerar:

(1) $H_{t}(j)$ é par, então $p_{t}(j)=0$ e

$$
\begin{gathered}
2^{t+1} \cdot\left(\frac{H_{t}(j)}{2}+\sum_{i \leq j} G_{t+1}(i)\right)+\sum_{s<t} p_{s}(j) \cdot 2^{s}+\sum_{s \leq t} G_{s}(j+1) \cdot 2^{s}+G_{t+1}(j+1) \cdot 2^{t+1}+0= \\
\left(2^{t} \cdot H_{t}(j)+\sum_{s<t} p_{s}(j) \cdot 2^{s}+\sum_{s \leq t} G_{s}(j+1) \cdot 2^{s}\right)+\sum_{i \leq j+1} G_{t+1}(i) \cdot 2^{t+1}
\end{gathered}
$$

E por hipótese de indução, isso é igual a

$$
2^{t} \cdot H_{t}(j+1)+\sum_{s<t} p_{s}(j+1) \cdot 2^{s}+\sum_{i \leq j+1} G_{t+1} \cdot 2^{t+1}
$$

Subcaso 1a: $H_{t}(j+1)$ é par, então $p_{t}(j+1)=0 \mathrm{e}$

$$
\begin{gathered}
2^{t+1}\left(\frac{H_{t}(j+1)}{2}+\sum_{i \leq j+1} G_{t+1}(i)\right)+\sum_{s<t} p_{s}(j+1) \cdot 2^{s}+0= \\
2^{t+1}\left(\left[\frac{H_{t}(j+1)}{2}\right]+\sum_{i \leq j+1} G_{t+1}(i)\right)+\sum_{s<t+1} p_{s}(j+1) \cdot 2^{s}
\end{gathered}
$$


E da definição de $\mathrm{H}$, isso é igual a

$$
2^{t+1} \cdot H_{t+1}(j+1)+\sum_{s<t+1} p_{s}(j+1) \cdot 2^{s} .
$$

Subcaso 1b: $H_{t}(j+1)$ é ímpar, então $p_{t}(j+1)=1 \mathrm{e}$

$$
\begin{gathered}
2^{t}\left(H_{t}(j+1)+\sum_{i \leq j+1} G_{t+1}(i) \cdot 2\right)+\sum_{s<t} p_{s}(j+1) \cdot 2^{s}= \\
2^{t}\left(2 \cdot\left[\frac{H_{t}(j+1)}{2}\right]+1+\sum_{i \leq j+1} G_{t+1}(i) \cdot 2\right)+\sum_{s<t} p_{s}(j+1) \cdot 2^{s}= \\
2^{t+1}\left(\left[\frac{H_{t}(j+1)}{2}\right]+\sum_{i \leq j+1} G_{t+1}(i)\right)+\sum_{s<t} p_{s}(j+1) \cdot 2^{s}+2^{t} .
\end{gathered}
$$

Da definição de $\mathrm{H}$ e como $p_{t}(j+1)=1$

$$
\begin{gathered}
2^{t+1} \cdot H_{t+1}(j+1)+\sum_{s<t} p_{s}(j+1) \cdot 2^{s}+p_{t}(j+1) \cdot 2^{t}= \\
2^{t+1} \cdot H_{t+1}(j+1)+\sum_{s<t+1} p_{s}(j+1) \cdot 2^{s} .
\end{gathered}
$$

(2) $H_{t}(j)$ é ímpar, então $p_{t}(j)=1$ e prova-se de modo análogo a (1) a igualdade desejada.

E tomando $t=\log (b)$ temos a função $\mathrm{F}$ desejada

$$
F(j)=2^{\log (b)} \cdot H_{\log (b)}(j)+\sum_{s<\log (b)} p_{s}(j) \cdot 2^{s} .
$$

A Contrapositiva do Teorema abaixo, o resultado principal desta seção, prova que, se vale uma versão de $\Delta_{0} P H P\left(\neg \exists a, F \in \Delta_{0}^{M}, F: 9 a \cdot \log (a) \longmapsto 8 a \cdot \log (a)\right)$ existem primos entre $a$ e $a^{11}$ (como $a$ é tão grande quanto quisermos, temos a infinidade dos primos). 
Teorema 3.6 Seja $a \in M$ e suponha que não existem primos em $M$ entre a e $a^{11}$. Então existe $F \in \Delta_{0}^{M}, F: 9 a \cdot \log (a) \longmapsto 8 a \cdot \log (a)$.

Demonstração: Primeiro note que por indução em $y \in M$, temos

$$
\exists z \leq y[\text { se } p \text { é o maior primo que divide y então } z \equiv 0 \bmod p
$$

e se $p<q$ são primos que dividem y e não existe primo $r, p<r<q$ que dividem $y$

$$
e z \equiv s \bmod p, \text { então } z \equiv s+1 \bmod q]
$$

Ainda mais, quaisquer tais dois $z$ serão congruentes mod $p$ para cada primo $p, p \mid y$. Consequentemente, podemos falar do número de primos que dividem $y, v(y)$, e para $i<v(y)$, do $i$-ésimo primo que divide $y$; e essas relações estão todas em $\Delta_{0}^{M}$. Também usando $I \Delta_{0}$ mostra-se que $v(y) \leq \log (y)$ para todo $y$.

Assumimos $a$ muito grande e que não existem primos em $M$ entre $a$ e $a^{11}$ e agora vamos definir um função $H \in \Delta_{0}^{M}$, com

$$
H: a \cdot \log \left(a^{10}\right) \longmapsto(1+[a / 2]) \cdot \log \left(a^{10}\right)+2 a \cdot \log (a) .
$$

A função $\mathrm{F}$ desejada decorre das propriedades de log, que valem em $\mathrm{M}$, e por $a$ ser um número muito grande.

$$
H: 10 a \cdot \log (a) \longmapsto(10(1+[a / 2])+2 a) \log (a) \leq(10+7 a) \log (a)<8 a \cdot \log (a) .
$$

Para $x<a \cdot \log \left(a^{10}\right)$ definimos $H(x)$ como segue: sejam $(i-1) \log \left(a^{10}\right) \leq x<$ $i \cdot \log \left(a^{10}\right)$ e $a^{10}+i=\prod_{j<v} p_{j}^{e_{j}}$, onde $v=v\left(a^{10}+i\right)$ e $p_{j}$ é o j-ésimo primo que divide $a^{10}+i$. Temos dois casos a considerar:

Caso 1: $\exists 0 \leq j<v$ tal que $\forall 1 \leq t \leq a, p_{j}^{e_{j}+1} \nmid a^{10}+t$ e $i$ é o menor número maior que zero tal que $p_{j}^{e_{j}} \mid a^{10}+i$.

Nesse caso tomamos o último tal $j$ e mapeamos $x$ usando a função linear

$$
y=2 a \cdot \log (a)+\left[\left(1+p_{j}\right) / 2\right] \log \left(a^{10}\right)+x-(i-1) \log \left(a^{10}\right)
$$

que leva o intervalo

$$
\left[(i-1) \log \left(a^{10}\right), i \cdot \log \left(a^{10}\right)\right]
$$


sobre

$$
\left[2 a \cdot \log (a)+\left[\left(1+p_{j}\right) / 2\right] \log \left(a^{10}\right), 2 a \cdot \log (a)+\left(1+\left[\left(1+p_{j}\right) / 2\right]\right) \log \left(a^{10}\right)\right] .
$$

Como $\prod_{j<v} p_{j}^{e_{j}}=a^{10}+i$ e $i<a$, temos $p_{j}<a^{11}$ e por hipótese não há primos entre $a$ e $a^{11}, \log 0 p_{j}<a$. Então,

$$
\left[\frac{1+p_{j}}{2}\right]<\left[\frac{1+a}{2}\right] \leq 1+\left[\frac{a}{2}\right] .
$$

Caso 2: Não vale o Caso 1 (aqui usaremos o Teorema 3.5 para construir as somatórias abaixo). Usando indução em $y=\prod_{i<\tau} q_{i}^{b_{i}}$, onde $q_{i}$ é o $i$-ésimo primo que divide $y$, temos

$$
\begin{gathered}
\log (y)=\sum_{i<\tau} b_{i} \cdot \log \left(q_{i}\right) \leq \sum_{i<\tau} b_{i}\left(1+\log \left(q_{i}\right)\right) \leq \sum_{i<\tau} b_{i}\left(\log \left(q_{i}\right)+\log \left(q_{i}\right)\right)=2 \log (y) \\
\log (y) \leq \sum_{i<\tau} b_{i}\left(1+\log \left(q_{i}\right)\right) \leq 2 \log (y)
\end{gathered}
$$

Então, sendo $j<v$ e $k<e_{j}$ tais que

$$
\begin{aligned}
& d_{0}=(i-1) \log \left(a^{10}\right)+\sum_{m<j} e_{m}\left(1+\log \left(p_{m}\right)\right)+k\left(1+\log \left(p_{j}\right)\right) \leq x< \\
& (i-1) \log \left(a^{10}\right)+\sum_{m<j} e_{m}\left(1+\log \left(p_{m}\right)\right)+(k+1)\left(1+\log \left(p_{j}\right)\right)=d_{1} .
\end{aligned}
$$

Tomamos $s, 1 \leq s \leq a$, tal que, para algum $b, p_{j}^{b} \mid a^{10}+s$ e $\forall 1 \leq t \leq a, p_{j}^{b+1} \nmid a^{10}+t$ e $\forall 1 \leq t<s, p_{j}^{b} \nmid a^{10}+t\left(p_{j}\right.$ do caso 1 vale para $\left.a^{10}+s\right)$. Agora temos dois subcasos à considerar:

Subcaso 2a. $s<i$.

Como $s<i, e_{j} \leq b$ e das hipóteses que $p_{j}^{e_{j}} \mid a^{10}+s$ e $p_{j}^{e_{j}} \mid a^{10}+i$, então $p_{j}^{e_{j}} \mid\left(a^{10}+i\right)-\left(a^{10}+s\right)=i-s$.

Seja $i-s=\prod_{m<\tau} q_{m}^{c_{m}}$, onde $q_{m}$ é o m-ésimo primo que divide $i-s$. Suponhamos $q_{r}=p_{j}$. Então, como $k<e_{j}$, temos $k+1 \leq e_{j} \mathrm{e}$

$$
\rho=2(i-s-1) \log (a)+\sum_{m<r} c_{m}\left(1+\log \left(q_{m}\right)\right)+(k+1)\left(1+\log \left(q_{r}\right)\right)
$$




$$
\leq 2(i-s-1) \log (a)+\sum_{m<r} c_{m}\left(1+\log \left(q_{m}\right)\right)+e_{j}\left(1+\log \left(q_{r}\right)\right)
$$

usando também $q_{r}=p_{j}$ e $p_{j}^{e_{j}} \mid i-s$, temos $e_{j} \leq c_{r}$ e

$$
\rho \leq 2(i-s-1) \log (a)+\sum_{m<\tau} c_{m}\left(1+\log \left(q_{m}\right)\right)
$$

e por $(*)$

$\rho \leq 2(i-s-1) \log (a)+2 \log (i-s) \leq 2(i-s-1) \log (a)+2 \log (a)=2(i-s) \log (a)$

e mapeamos linearmente o intervalo $\left[d_{0}, d_{1}\right)$ no intervalo com extremos

$$
\begin{gathered}
2(i-s-1) \log (a)+\sum_{m<r} c_{m}\left(1+\log \left(q_{m}\right)\right)+k\left(1+\log \left(q_{r}\right)\right), \\
2(i-s-1) \log (a)+\sum_{m<r} c_{m}\left(1+\log \left(q_{m}\right)\right)+(k+1)\left(1+\log \left(q_{r}\right)\right) .
\end{gathered}
$$

É importante notar que o último intervalo determina $q_{r}$ e $k$, consequentemente $s$ e $i$ e por fim $\left[d_{0}, d_{1}\right)$.

Subcaso 2b. $i<s$. Note primeiro que o maior $i$ (com $k$ fixado) para o qual o Subcaso 2a pode ser aplicado é

$$
s+\left[\frac{a-s}{p_{j}^{k}}\right] \cdot p_{j}^{k} .
$$

Nesse caso, procedemos como no Subcaso 2a, lembrando que $i<s$ e substituindo $i-s$ por

$$
s-i+\left[\frac{a-s}{p_{j}^{k}}\right] \cdot p_{j}^{k}\left(>s+\left[\frac{a-s}{p_{j}^{k}}\right] \cdot p_{j}^{k}-s=i-s\right) \text {, para } i \text { máximo do Subcaso 2a. }
$$

Novamente o intervalo que mapeamos $\left[d_{0}, d_{1}\right)$ irá determinar $p_{j}$ e $k$, consequentemente $s$ e por fim $i$ e $\left[d_{0}, d_{1}\right)$.

Com isso vemos que $H$ é injetora e como ela é claramente $\Delta_{0}^{M}$ temos o desejado.

Como consequência, usando o Corolário 3.1, temos que de $I \Delta_{0}$ e de $\exists x^{\log (x)}$ para todo $x$ decorre a infinitude dos primos. 
Teorema $3.7 I \Delta_{0} \vdash \forall x\left(\exists x^{\log (x)} \longrightarrow \exists y>x(y\right.$ primo $\left.)\right)$.

Decorre do Corolário 3.1 e da contrapositiva do Teorema 3.6.

Terminamos este capítulo enunciando outro teorema interessante que se relaciono com os resultados aqui apresentados.

Teorema 3.8 (Teorema de Sylvester) Da teoria $I \Delta_{0}+\forall x\left(\exists x^{\log (x)}\right)$ deduz-se o Teorema de Sylvester, que diz que se $1 \leq x<y$ então algum entre os seguintes números $y+1, y+2, \ldots, y+x$ tem um divisor primo $p>x$.

Veja a tese de doutoramento de Alan R. Woods, [8, Capítulo 1, §3, pp. 23-36], para demonstração detalhada. 


\section{Capítulo 4}

\section{Conclusão}

Aqui são mostrados passos interessantes na área conhecida como Bounded Reverse Mathematics, cujo objetivo é formalizar e provar versões discretas de teoremas matemáticos em teorias fracas da Aritmética Limitada. Entre eles uma definição $\Delta_{0}$ de $x^{y}=z$, o Teorema Limitado da Casa dos Pombos e a Infinidade dos Primos. E ainda mais, a relação entre essas ideias, pois ambas decorrem de $I \Delta_{0}+\forall x>1\left(\exists x^{\log (x)}\right)$, como visto no capítulo 2 .

Quanto a caminhos futuros, temos problemas abertos com relação muito clara com os discutidos na presente dissertação, como os seguintes.

Problema Aberto 1 PHP é realmente necessário para provar a infinidade de primos, ou seja, $\forall x \exists y>x(y$ primo) implica alguma forma fraca de PHP?

Problema Aberto $2 I \Delta_{0}+(\forall x \exists y>x(y$ primo $))$ implica a existência de alguma função $\left(\Delta_{0}\right.$ ou $\left.\Delta_{1}\right)$ que seja superpolinomial (uma função é dita superpolinomial se o tempo de execução de seu algoritmo não pode ter um polinômio como limitante superior)?

Os problemas 1 e 2 podem trazer alguma luz também na Teoria dos Números, mostrando possibilidades ou restrições nos chamados métodos de crivos (sieve methods). A prova original da infinidade de primos de Euclides usa o fatorial de um número, uma função de crescimento exponencial. Aquela apresentada nesta dissertação usa uma função de crescimento subexponencial, mas ainda superpolinomial. Se o Problema 1 tiver solução positiva, seria um resultado surpreendente.

Em uma outra direção, mas muito importante: 
Problema Aberto $3 I \Delta_{0}$ é finitamente axiomatizável? Esta questão tem implicações importantes em Complexidade Computacional (ver, por exemplo, [3, Capítulo V, Corolário 4.39, p. 353]). Os especialistas na área acreditam que este problema tem solução negativa, pois ele implica o colapso da classe de complexidade chamada PH (Polynomial Hierarchy).

Um outro problema interesante surgiu quando W. Hesse, provou recentemente em [4], que a relação exponenciação modular de números de $O(\log (n))$ bits (o tempo de execução do algoritmo para $n$ grande é no máximo proporcional a log $(n))$ é definível em primeira ordem; com isso prova-se que $x^{y} \equiv z(\bmod n)$ é definível em $\Delta_{0}$. No entanto, a prova disso depende do Pequeno Teorema de Fermat (se p for primo e a inteiro, então $\left.a^{p}=a(\bmod p)\right)$, assim não é claro se as propriedades básicas da exponenciação modular são demonstráveis em $I \Delta_{0}$. E a pergunta que surge é:

Problema Aberto 4 Encontrar uma definição $\Delta_{0}$ de $x^{y} \equiv z(\bmod n)$, tal que as propriedades básicas $x^{y} \cdot x^{z} \equiv x^{y+z}(\bmod n)$ e $\left(x^{y}\right)^{z} \equiv x^{y \cdot z}(\bmod n)$ sejam demonstráveis em $I \Delta_{0}$.

Enfim, esses problemas dão um pequeno vislumbre da amplitude da área de pesquisa em Aritmética. 


\section{Referências Bibliográficas}

[1] Atserias A., Improved Bounds on the Weak Pigeonhole Principle and Infinitely Many Primes form Weaker Axioms, MFCS 2001, pp 148-158. Theoretical Computer Science Vol. 295, Issues 1-3, (2003), pp. 27-39.

[2] Dimitracopoulos, C. e Gaifman, H., Fragments of Peano's Arithmetic and the MRDP Theorem, Logic and Algorithmic, Geneve 1982, Monographie No. 30 de L'Enseignement Mathematique (1982), pp. 187-206.

[3] Hájek P. e Pudĺak P., Metamathematics of First-Order Arithmetic, Perspectives in Mathematical Logic, Volume 3. Berlin: Springer-Verlag 1998.

[4] Hesse W., Division is in Uniform $\mathrm{TC}^{\circ}$, in Proceedings of the 28th International Colloquium On Automata, languages and Programming, Lecture Notes in Computer Science, Vol. 2076 (2001), pp 104-114.

[5] Nguyen P., Proving Infinitude of Prime Numbers Using Binomial Coeficients, 22nd International Workshop, CSL 2008, 17th Annual Conference of the EACSL, Bertinoro, Italy, September 16-19, 2008. Proceedings. Lecture Notes in Computer Science, Vol.5213 (2008), pp 184-198.

[6] Paris J. B. e Kirby L., $\Sigma_{n}$-collection schemes in arithmetic, Logic Colloquim 'ry', North-Holland, Amsterdam, 1978, pp. 199-209.

[7] Paris J. B., Wilkie A. J. e Woods A. R., Provability of the Pigeonhole Principle and the Existence of Infinitely Many Primes, The Journal of Symbolic Logic, Vol. 53, No. 4 (Dec., 1988), pp. 1235-1244.

[8] Woods, A. R., Some Problems in Logic and Number Theory, and Their Connections, Tese de Doutorado em Filosofia na Universidade de Manchester, 1981. 
[9] Woods A. R. e Cornaros C., On Bounded Arithmetic Augmented by the Ability to Count Certain Sets of Primes, The Journal of Symbolic Logic Vol. 74, No. 2 (Jun., 2009), pp. 455-473. 


\section{Índice Remissivo}

$$
\begin{aligned}
& B \Sigma_{1} 10 \\
& F: a \longmapsto b, 2 \\
& G_{y, w}, 21 \\
& H_{c}^{d}: \subseteq 2^{d} \longrightarrow a, 13 \\
& I \Delta_{0}, 5 \\
& L=\{0,1,+, \cdot, \leq\}, 5 \\
& M, 3 \\
& M \models I \Delta_{0}, 9 \\
& M \prec_{0} K, 10 \\
& M \subset_{e} K, 10 \\
& \Delta_{0}, 5 \\
& \Delta_{0} P H P, 2 \\
& \Sigma_{1}, 10 \\
& \delta(u, v, w, i), 6 \\
& (\epsilon+1) x, 2 \\
& \log (a)^{k}, 20 \\
& \log (x), 2 \\
& \log (x), 12 \\
& \sigma * \tau: b \longrightarrow 2,13 \\
& \sigma: d \longmapsto 2,13 \\
& \unlhd, 9 \\
& a \dot{-} b, 14 \\
& c \oplus d=e, 16 \\
& c \otimes d=e, 16 \\
& p(i, u, v), 6 \\
& q_{j}^{*} c=e, 16 \\
& r m(u, v)=z, 6 \\
& v(y), 25 \\
& , 33 \\
& {[\mathrm{x}], 22} \\
&
\end{aligned}
$$

